\title{
Aspiration thrombectomy and histopathologic examination of thrombus for early identification of embolic myocardial infarction
}

\author{
Maciej T. Wybraniec ${ }^{1}$, Edyta Reichman-Warmusz ${ }^{2}$, Michał Lelek ${ }^{1}$, Tomasz Bochenek ${ }^{1}$, Romuald J. Wojnicz ${ }^{2}$, \\ Katarzyna Mizia-Stec ${ }^{1}$ \\ ${ }^{1}$ First Department of Cardiology, School of Medicine in Katowice, Medical University of Silesia, Poland \\ ${ }^{2}$ Department of Histology, School of Medicine with the Division of Dentistry in Zabrze, Medical University of Silesia in Katowice, Poland
}

Adv Interv Cardiol 2019; 15, 4 (58): 489-491

DOI: https://doi.org/10.5114/aic.2019.90225

The clinical differentiation between thrombophilia-related coronary embolization and classic atheroma-related acute myocardial infarction (AMI) remains challenging as laboratory tests may be unreliable in the acute setting [1]. Since angiographic and intravascular imaging is often inconclusive, we propose the use of pathological examination of the aspirated thrombus for selection of patients requiring chronic anticoagulation in addition to antiplatelet therapy. This concept has recently been adopted in a 37-year-old patient presenting with 2-hour retrosternal chest pain at rest in the course of inferior wall ST-segment elevation AMI. Pre-procedural transthoracic echocardiography (TTE) showed mildly depressed left ventricular (LV) systolic function with hypokinesis of the inferior wall and presence of a well-organized thrombus attached to apical segments of LV $(28 \times 21 \mathrm{~mm})$ (Figure $1 \mathrm{~A})$. The coronary angiography performed via a right radial approach showed acute occlusion of the right coronary artery and non-significant, parietal lesions within the left coronary artery. The occlusion was crossed with a Balance Middleweight guide wire (Figure $1 \mathrm{~B}$ ) and the thrombus was aspirated using an Export thrombectomy catheter (Figure 1 C). The aspirated thrombus (Figure $1 \mathrm{D}$ ) was then stored in neutral buffered formalin and PolyTransport buffer. Subsequently, a $3.5 \times 16 \mathrm{~mm}$ Promus Element stent was implanted in the lesion and post-dilated with a $4.0 \times 15 \mathrm{~mm}$ non-compliant balloon, leading to complete restoration of the patency of the vessel with a small distal residual thrombus (Figure $1 \mathrm{E}$ ). Prolonged ECG monitoring showed no proof of atrial fibrillation. The histopathologic examination, which comprised standard hematoxylin and eosin staining, showed a complex structure, characterized by hypocellular retracted fibrin con- glomerate, partially infiltrated with neutrophils (Figures $1 \mathrm{~F}$ and $\mathrm{G}$ ). The image was consistent with a well-organized, relatively old thrombus, which did not correspond with in situ clot formation due to rupture of the atheromatous plaque. In addition to aspirin and ticagrelor and intra-procedural bolus of unfractionated heparin, the patient received transient 18-hour infusion of eptifibatide, followed by intravenous infusion of unfractionated heparin overlapping with initiation of oral anticoagulation. At post-procedural day 3 , the patient was switched from ticagrelor to clopidogrel. The patient was discharged home at post-procedural day 6 with clopidogrel, aspirin and warfarin. The extended laboratory tests at 6 weeks confirmed the presence of a prothrombin heterozygous G20210A mutation responsible for the hypercoagulable state. The follow-up TTE confirmed only partial resolution of the thrombus. At 6-month follow-up aspirin was withdrawn, while clopidogrel was recommended up to 12 months, followed by life-long treatment with a vitamin $\mathrm{K}$ antagonist.

Despite unfavourable results of recent randomized controlled trials [2], the use of aspiration thrombectomy might potentially facilitate early diagnosis of thromboembolic AMI by means of early histopathologic examination of the aspirated thrombus, advocating in favour of initiation of chronic anticoagulation therapy during index hospitalization [3]. This approach, however, is limited by the absence of intracoronary imaging in the form of optimal coherence tomography (OCT), which could delineate the absence of intimal rupture, while the older age of the thrombus might also be related to episodes of prior subclinical non-occlusive coronary thrombosis [4]. Still, the presence of $\mathrm{LV}$ thrombus and the intracoronary thrombus 

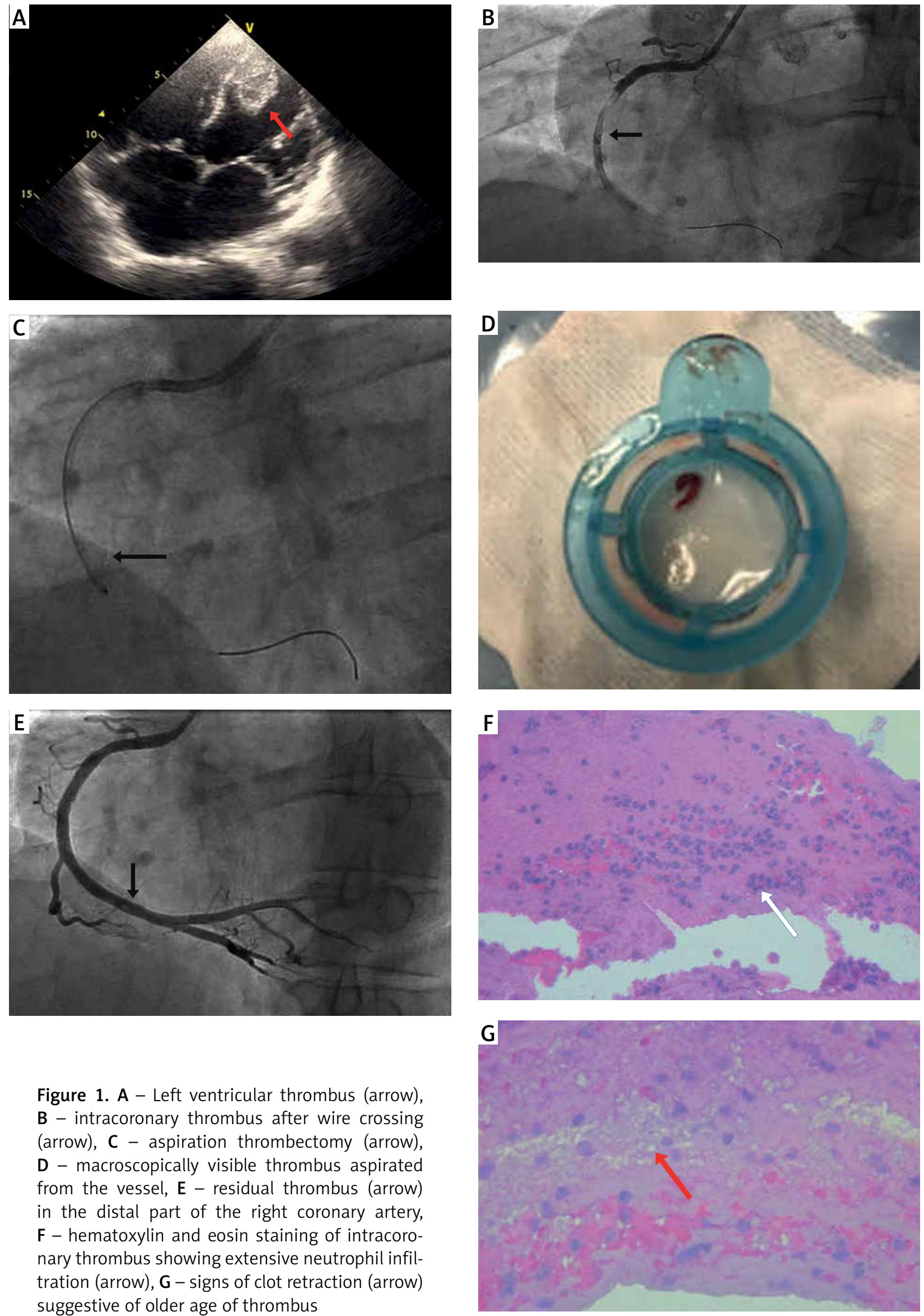

Figure 1. A - Left ventricular thrombus (arrow), B - intracoronary thrombus after wire crossing (arrow), C - aspiration thrombectomy (arrow), D - macroscopically visible thrombus aspirated from the vessel, E - residual thrombus (arrow) in the distal part of the right coronary artery, $\mathbf{F}$ - hematoxylin and eosin staining of intracoronary thrombus showing extensive neutrophil infiltration (arrow), G - signs of clot retraction (arrow) suggestive of older age of thrombus 
composition should advocate in favour of embolic aetiology of AMI and guide appropriate treatment.

\section{Acknowledgments}

This work was financed from the Grant for Young Scientists under the auspices of Club 30 of the Polish Cardiac Society.

\section{Conflict of interest}

The authors declare no conflict of interest.

\section{References}

1. Shibata T, Kawakami S, Noguchi T, et al. Prevalence, clinical features, and prognosis of acute myocardial infarction attributable to coronary artery embolism. Circulation 2015; 132: 241-50.

2. Jolly SS, Cairns JA, Yusuf S, et al.; TOTAL Investigators. Randomized trial of primary $\mathrm{PCl}$ with or without routine manual thrombectomy. N Engl J Med 2015; 372: 1389-98.

3. Carol A, Bernet M, Curós A, et al. Thrombus age, clinical presentation, and reperfusion grade in myocardial infarction. Cardiovasc Pathol 2014; 23: 126-30.

4. Kostić J, Orlić D, Borović ML, et al. Coronary thrombi neovascularization in patients with ST-elevation myocardial infarction clinical and angiographic implications. Thromb Res 2014; 134: 1038-45. 